\title{
METICILLINO-RESISTENZA
}

IN CEPPI DI STAPHYLOCOCCUS AUREUS

DI ORIGINE ALIMENTARE E ANIMALE

\section{METHICILLIN-RESISTANT STAPHYLOCOCCUS AUREUS STRAINS IN FOOD AND ANIMAL}

\author{
Traversa A. ${ }^{1}$, Bianchi D.M. ${ }^{1}$, Parlato C. ${ }^{2}$, Fossati L. ${ }^{2}$, Cavallerio P. ${ }^{2}$, Domenis L. ${ }^{3}$, Spedicato R. ${ }^{3}$, \\ Battisti A. ${ }^{4}$, Decastelli L. ${ }^{1}$ \\ (1) NRL Stafilococchi coagulasi positivi, compreso S. aureus, Torino \\ (2) Ospedale San Giovanni Battista, Laboratorio di Microbiologia, Torino \\ $\left({ }^{3}\right)$ Centro di Referenza per le Malattie degli Animali Selvatici, IZS Piemonte, Liguria, Valle d'Aosta, Sezione di Aosta \\ (4) Centro di Referenza Nazionale per l'Antibioticoresistenza, IZS Lazio e Toscana, Roma
}

SUMMARY

Key words

\begin{abstract}
Some authors reported the possibility of a methicillin-resistant Staphylococcus aureus (MRSA) human infections from meat and dairy products and methicillin-resistant Staphylococcus intermedius isolation in animals. The aim of this study is to investigate the methicillin-resistance in $S$. aureus strains and in $S$. intermedius strains (food and wild animals). 236 S.aureus strains from food, 36 S.aureus strains and 1 S. intermedius strain from wild animals were analyzed. 2 (0.74\%) MRSA strains from bovine milk were phenotipically resistant to cefoxitin, grew on chromogenic medium (MRSA Brilliance Oxoid) and were mecA positive. All MRSA strains had the spa-type t899. All mecA positive strains showed at least resistance to eight of the antibiotics tested but none to glicopeptides. Both MRSA strains were enterotoxigenic.
\end{abstract}

MRSA, MRSI, dairy products, wild animals, mecA gene, spa-Typing

\section{INTRODUZIONE}

Staphylococcus aureus è comunemente isolato a livello cutaneo e nasofaringeo nel $30 \%$ della popolazione umana, senza causare malattia. Analogo comportamento possiede in campo veterinario Staphylococcus intermedius, presente nei carnivori domestici a livello delle giunzioni mucocutanee e nella cavità oronasale. Nei soggetti immunocompromessi, tuttavia, $S$. aureus è responsabile di gravi infezioni soprattutto a livello nosocomiale e l'acquisizione di proprietà di resistenza antibiotica ha contribuito ad aumentare la patogenicità di alcuni ceppi. L'acquisizione di infezioni da MRSA è generalmente di tipo ospedaliero (HA-MRSA), sono stati, tuttavia, isolati ceppi meticillino-resistenti in soggetti immunocompetenti non ospedalizzati e in piccole comunità chiuse (caserme, scuole, carceri e case di riposo); tale evento è definito "community-acquired MRSA" (CA-
MRSA). Alcuni autori sottolineano il rischio di una infezione anche per via alimentare (FBA-MRSA), in particolare modo nel settore suinicolo. Gruppi di ricercatori hanno riportato casi umani di infezione in soggetti a rischio (veterinari, allevatori) in contatto con suini, cavalli e cani. Tali isolamenti, a livello europeo, nei suini e in diverse specie animali hanno evidenziato la presenza del complesso clonale ST398 (1). La distinzione epidemiologica corrisponde a differenze genotipiche: il gene mecA, responsabile della meticillino-resistenza, è veicolato da un elemento genetico mobile integrato nel genoma batterico definito "staphylococcal cassette chromosome mec" (SCC-mec) che si presenta di tipo I, II e III negli HAMRSA e di tipo IV e V nei CA-MRSA. Le infezioni "comunitarie" determinano frequentemente maggiore patogenicità e mortalità grazie alla produzione della leucocidina di Panton Valentine (PVL) indotta dalla presenza del gene omonimo, che provoca ne- 
crosi tissutale a livello cutaneo e polmonare. La meticillino-resistenza nei CA-MRSA è generalmente crociata e limitata ai betalattamici e ai carbapenemi, mentre negli HA-MRSA si accompagna spesso a resistenza nei confronti di altri antibiotici.

La pubblicazione, inoltre, del parere EFSA (27 marzo 2009) (2) relativo alla valutazione del significato per la salute pubblica degli MRSA negli animali e negli alimenti rappresenta un valido strumento per affrontare tale problematica.

Recentemente in Europa si sono registrati isolamenti di Staphylococcus intermedius meticillino-resistente (MRSI) "mecA positivo" a partire da tamponi cutanei di cani e gatti. La trasmissione all'uomo da animali d'affezione è stata dimostrata in Olanda (3).

\section{MATERIALI E METODI}

Sono stati sottoposti a ricerca di meticillino-resistenza 273 ceppi: 236 ceppi di $S$. aureus da alimenti di origine animale, 36 ceppi di $S$. aureus e 1 ceppo di $S$. intermedius da animali selvatici. I campionamenti sono stati effettuati nel periodo gennaio-agosto 2008 durante l'attività di Controllo Ufficiale sugli alimenti (grafico 1) e, per quanto riguarda i selvatici, nel periodo 2003-2009 presso il Centro di Referenza per le Malattie degli Animali Selvatici (Ce.R.M.A.S) (grafico 2).

I ceppi isolati, identificati e stoccati in cryobank (Mast Diagnostic) a $-18^{\circ} \mathrm{C}$ secondo le indicazioni fornite dalla Ditta produttrice, sono stati rivitalizzati mediante passaggio su piastra di agar sangue.
Allo scopo di valutare l'acquisizione di proprietà meticillino-resistenti si è proceduto alla semina su terreno cromogeno (MRSA Brilliance - Oxoid) e all'esecuzione del test di diffusione in Mueller-Hinton agar con dischetto di cefoxitina $30 \mathrm{~g}$, secondo le linee guida del Clinical and Laboratory Standard Institute (CLSI Gennaio 2008), utilizzando una sospensione batterica in acqua sterile pari a $0.5 \mathrm{Mc}-$ Farland. Il ceppo MRSA (ATCC 49775) è stato utilizzato quale controllo positivo.

I ceppi risultati "resistenti" sul terreno cromogeno (crescita di colonie tipiche) e al test di diffusione in agar (alone di inibizione $\leq 21 \mathrm{~mm}$ ) sono stati sottoposti ad estrazione del DNA (Ultraclean microbial dna solution kit - MO BIO Laboratories) per la ricerca dei geni mec e ccr (determinazione della Staphylococcal Chromosome Cassette mec o SCCmec) secondo il protocollo operativo descritto da Zhang et al. (PCR Multiplex)(4).

I ceppi mecA positivi sono stati sottoposti alla ricerca dei geni codificanti la produzione della Leucocidina di Panton Valentine tramite PCR end-point secondo il protocollo descritto da Lina et al. (5), al sequenziamento del gene spa (spa-typing) e alla determinazione del profilo di resistenza mediante $M i$ croscan.

\section{RISULTATI}

Tra i ceppi di $S$. aureus analizzati nel corso della ricerca, $8(2.94 \%)$ sono cresciuti sul terreno cromogeno MRSA Brilliance; di questi solo $2(0.74 \%)$ isolati da latte di massa bovino e da organi di suino sono ri-

Grafico 1: ceppi di S. aureus $(\mathrm{n}=236)$ da alimenti di origine animale

\section{$65 \%$}

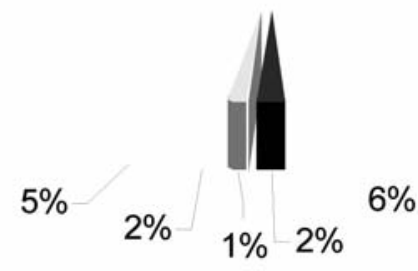

$12 \%$

\section{$7 \%$}

- latte vaccino

- formaggi latticini

- preparazioni a base di carne lagomorfi e selvaggina allevata
- latte ovi-caprino

- carni bovine, suine e ovicaprine pasta alimentare non noto 
Grafico 2: ceppi di $S$. aureus $(\mathrm{n}=36)$ isolati da animali selvatici

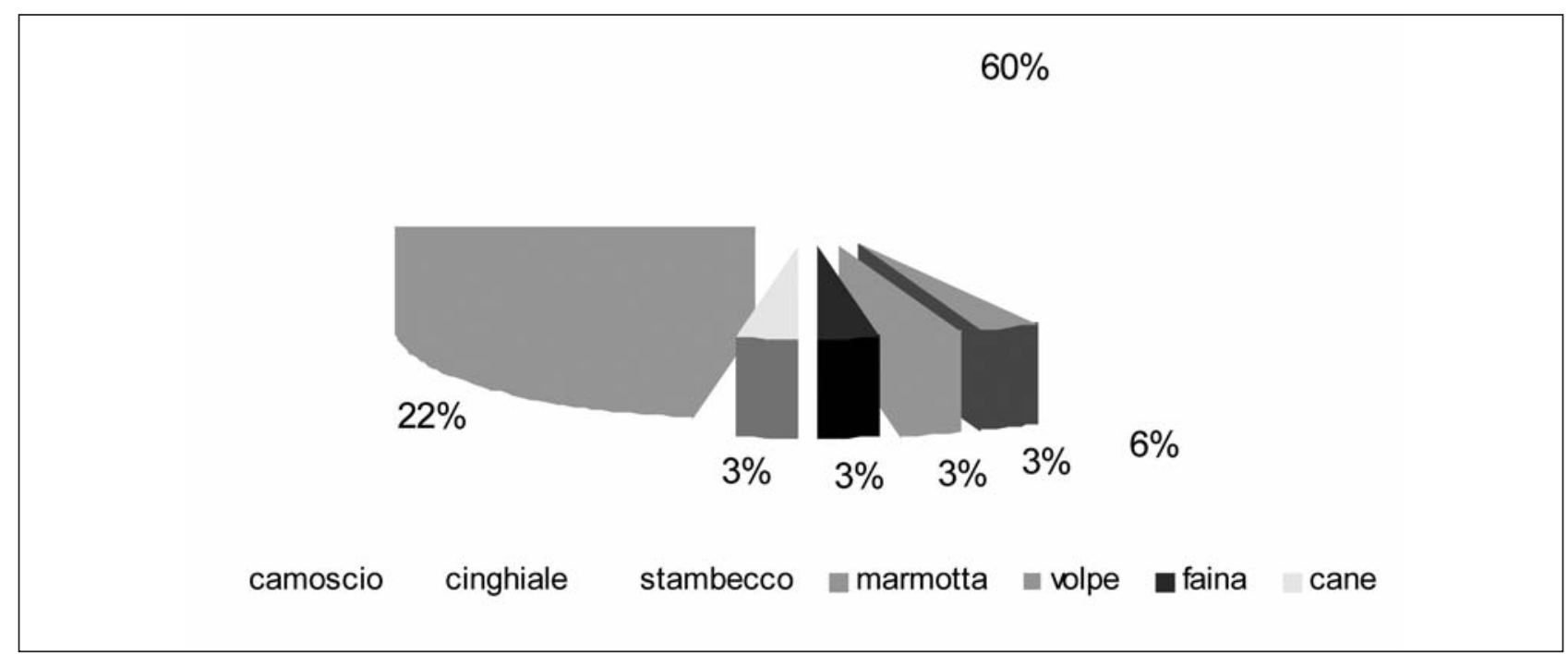

sultati meticillino-resistenti al test della cefoxitina con presenza del gene mecA (cassetta cromosomica: SCC-mec di tipo IV e V; spa-type t899); la ricerca della leucocidina di Panton Valentine è risultata negativa. I rimanenti 6 ceppi (2\%) cresciuti sul terreno cromogeno sono però risultati meticillino-sensibili (PCR: assenza del gene mecA; spa-type t091).

$\mathrm{Nel}$ corso della ricerca è stato inoltre testato un ceppo di $S$. intermedius isolato da una volpe; tale ceppo non è cresciuto sul terreno cromogeno e si è dimostrato sensibile al test di diffusione in agar con cefoxitina.

I risultati ottenuti, relativi ai ceppi MRSA, sono sintetizzati nella tabella 1.

I ceppi mecA-positivi hanno manifestato multiresistenza nei confronti di almeno otto antibiotici tra quelli utilizzati, mentre sono risultati sensibili ai gli- copeptidi. Entrambi i ceppi MRSA sono risultati resistenti a betalattamici, tetraciclina e clindamicina e sensibili ai chinoloni, gentamicina e cloramfenicolo; il ceppo isolato da latte bovino ha presentato resistenza anche nei confronti di claritromicina, eritromicina e trimetoprim/sulfadiazina (tabella 2).

\section{CONSIDERAZIONI E CONCLUSIONI}

La crescita su terreno cromogeno ha evidenziato 6 positivi in più rispetto al test di diffusione in agar con cefoxitina (Se 99.9\%; Sp 97.8\%) e difficoltà nella definizione di "resistente". Tale dicitura, legata alla crescita di colonie color "blue denim", si è rivelata talvolta difficilmente interpretabile per la presenza di colonie con tonalità di colore differenti risultate

Tabella 1: caratteristiche biochimiche e biomolecolari dei ceppi MRSA

\begin{tabular}{|c|c|c|}
\hline Specie & S. aureus & S. aureus \\
\hline Matrice & organi suino & latte di massa vaccino \\
\hline Enterotossina & POS & POS \\
\hline MRSA Brilliance Oxoid & POS & POS \\
\hline Test di diffusione in agar con Cefoxitina & $13 \mathrm{~mm}$ & $>2 \mu \mathrm{m} / \mathrm{ml}$ \\
\hline Mic Oxacillina & $>2 \mu \mathrm{g} / \mathrm{ml}$ & POS \\
\hline PCR $m e c A$ & POS & tipo IV \\
\hline SCC-mec & tipo V & NEG \\
\hline Leucocidina PV & NEG & t899 \\
\hline spa-typing & t899 & \\
\hline
\end{tabular}


Tabella 2: Profili di resistenza dei ceppi mecA +

\begin{tabular}{|c|c|c|}
\hline & S. aureus da organi di suino & S. aureus da latte bovino \\
\hline Penicillina & $\mathbf{R}$ & $\mathbf{R}$ \\
\hline Ampicillina & $\mathbf{R}$ & $\mathbf{R}$ \\
\hline Amoxicillina / Acido clavulanico & $\mathbf{R}$ & $\mathbf{R}$ \\
\hline Oxacillina & $\mathbf{R}$ & $\mathbf{R}$ \\
\hline Cefalotina & $\mathbf{R}$ & $\mathbf{R}$ \\
\hline Cefuroxime & $\mathbf{R}$ & $\mathbf{R}$ \\
\hline Teicoplanina e Vancomicina & $\mathrm{S}$ & $\mathrm{S}$ \\
\hline Gentamicina & $\mathrm{S}$ & $\mathrm{S}$ \\
\hline Eritromicina & $\mathrm{S}$ & $\mathbf{R}$ \\
\hline Claritromicina & $\mathrm{S}$ & $\mathbf{R}$ \\
\hline Tetraciclina & $\mathbf{R}$ & $\mathbf{R}$ \\
\hline Ciprofloxacina & $\mathrm{S}$ & $\mathrm{S}$ \\
\hline Levofloxacina & $\mathrm{S}$ & $\mathrm{S}$ \\
\hline Ofloxacina & $\mathrm{S}$ & $\mathrm{S}$ \\
\hline Clindamicina & $\mathbf{R}$ & $\mathbf{R}$ \\
\hline Cloramfenicolo & $\mathrm{S}$ & $\mathrm{S}$ \\
\hline Trimetoprim / Sulfadiazina & $\mathrm{S}$ & $\mathbf{R}$ \\
\hline
\end{tabular}

sensibili al test di diffusione in agar.

La percentuale di MRSA riscontrata $(0.74 \%)$ ricalca i risultati ottenuti in altri studi che riportano valori intorno al $3.75 \%$ da latte vaccino e formaggi latticini (6) e allo $0.5 \%$ da carne di pollo (7). La possibile sottostima può essere dovuta alla ricerca del gene mecA sui ceppi risultati resistenti al test di diffusione su agar: alcuni autori riportano la possibilità che ceppi mecA-positivi possano dimostrarsi fenotipicamente sensibili ai betalattamici. Tale proprietà può essere spiegata dalla "eteroresistenza" dei ceppi, la capacità di alcuni cloni batterici di esprimere fenotipicamente la resistenza (6).

Due ceppi MRSA isolati da latte bovino e da organi di suino presentano, rispettivamente, una cassetta cromosomica di tipo IV e di tipo V e spa-type t899; tale riscontro appoggia l'ipotesi che possa trattarsi di ceppi MRSA "comunitari" di origine animale. Prendendo in considerazione i dati bibliografici disponibili, ceppi con spa-type t899 isolati da carne suina, di pollo, tacchino e faraona, sottoposti a MLST (multilocus sequence type), risultano appartenere al Sequence Type 398 (7). Secondo il gruppo scientifico di esperti sui pericoli biologici dell'EFSA (BIOHAZ) i dati ottenuti mediante spa-typing possiedono ampia congruenza con i risultati del metodo MLST e di altre tecniche di tipizzazione (2). Si può presupporre che i due ceppi riscontrati possano essere ricondotti al sopraccitato complesso clonale.

Ceppi MRSA con SCC-mec di tipo IV e V presen- tano nell'uomo un profilo di resistenza circoscritto ai betalattamici. I due ceppi MRSA evidenziati nello studio manifestano resistenza nei confronti di altre classi di antibiotici; tale rilievo induce a riflettere sul ruolo e sulla gestione degli antibiotici in stalla, forse troppo utilizzati come ausilio terapeutico e come promotori di crescita.

L'assenza di isolamento di ceppi MRSA da animali selvatici offre una nota ottimistica al panorama medico e veterinario.

Secondo il parere EFSA del 27 marzo 2009 i ceppi MRSA possiedono comunemente i geni che codificano la produzione delle enterotossine: a oggi è stato registrato un solo caso di intossicazione alimentare. Parimenti non vi è evidenza che il rischio di infezione sia maggiore a seguito di contatto o consumo di alimenti contaminati da ceppi MRSA (compreso il Sequence Type 398). Il consumo e la manipolazione di alimenti contaminati sono da considerare potenziali veicoli di trasmissione di MRSA. Viene quindi raccomandato lo sviluppo di un sistema di sorveglianza e monitoraggio nell'uomo e negli alimenti al fine di valutare l'andamento della diffusione ed evoluzione dei ceppi MRSA di origine animale (2). 


\section{BIBLIOGRAFIA}

1) Huijdens X.W., van Dijke B.J., Spalburg E., van Santen-Verheuvel M.G., Heck M.E., Pluister G.N., Voss A., Wannet W.J., de Neeling A.J. (2006) "Community-acquired MRSA and pig-farming” Annals of Clinical Microbiology and Antimicrobials . Nov 10 5:26;

2) European Food Safety Authority (2009) “Assessment of the Public Health significance of meticillin resistant Staphylococcus aureus (MRSA) in animals and foods" The EFSA Journal 993, 1-73;

3) van Duijkeren E., Houwers D.J., Schoormans A., Broekhuizen-Stins M.J., Ikawaty R., Fluit A.C., Wagenaar J.A. (2008) "Transmission of methicillin-resistant Staphylococcus intermedius between humans and animals" Veterinary Microbiology Vol. 128, Issues 1-2, 1 April, p.213215 ;

4) Zhang K., McClure J.A., Elsayed S., Louie T., Conly J.M. (2005) "Novel Multiplex PCR Assay for Characterization and Concomitant Subtyping of Staphylococcal Cassette Chromosome mec Types I to V in Methicillin-Resistant Staphylococcus aureus" Journal of Clinical Microbiology, Vol. 43, No. 10, p. 5026-5033;

5) Lina G., Piemont Y., Godail-Gamot F., Bes M., Peter M.O., Gauduchon V., Vandenesch F. Etienne J. (1999) "Involvement of Panton-Valentine Leukocidin-Producing Staphylococcus aureus in Primary Skin Infections and Pneumonia" Clinical Infectious Diseases 29:1128-32;

6) Normanno G., Corrente M., La Salandra G., Dambrosio A., Quaglia N.C., Parisi A., Greco G., Bellacicco A.L., Virgilio S., Celano G.V. (2007) "Methicillin-resistant Staphylococcus aureus (MRSA) in foods of animal origin product in Italy" International Journal of Food Microbiology, 117 219-222G;

7) de Boer E., Zwartkruis-Nahuis J.T.M., Wit B., Huijsdens X.W., de Neeling A.J., Bosch T., van Oosterom R.A.A., Vila A., Heuvelink A.E. (2009) "Prevalence of methicillinresistant Staphylococcus aureus in meat" International Journal of Food Microbiology, doi: 10.1016/j.ijfoodmicro.2008.12.007.

Finanziamento Ricerca corrente 2007: "Isolamento e identificazione di S.aureus meticillino-resistente

(CA-MRSA) negli alimenti e nell'uomo: problematica emergente in sanità pubblica" 